\title{
La formación profesional de hostelería y turismo en la localidad española de Ciudad Real'
}

\author{
Cecilia Ruiz Esteban \\ Doctora en Psicología. \\ Universidad de Murcia. España. \\ Correo electrónico: cruiz@um.es \\ Inmaculada González García \\ Máster Universitario en Formación del Profesorado de Educación Secundaria \\ Universidad de Murcia. España \\ Correo electrónico: inmaculada.gonzalez7@um.es
}

Recibido: $16 / 05 / 2020$

Evaluado: $21 / 05 / 2020$

Aceptado: 15/07/2020

\section{Resumen}

Pese al papel fundamental que la reforma educativa española otorga a la Formación Profesional se desconoce si la percepción de la sociedad ha mejorado. Para poder intentar mejorar la imagen social de la Formación Profesional debemos conocerla. Aunque la investigación es escasa, existen algunos estudios que destacan la valoración positiva que la sociedad le otorga a la vinculación entre formación y empresa de este tipo de educación. En este sentido, el objetivo de este estudio es valorar la percepción de los estudiantes de Formación Profesional sobre las expectativas de futuro que les ofrece esta etapa formativa. Los participantes fueron 181 estudiantes de Formación Profesional de la Especialidad de Hostelería y Turismo (41.7\% fueron varones). Se utilizó el Cuestionario de Perspectivas de Futuro de Husman y Shell (2008) y un cuestionario creado ad hoc para recoger los datos sociodemográficos. Se realizaron pruebas $t$ de Student para analizar las diferencias de medias entre distintas variables sociodemográficas en las cuatro dimensiones del cuestionario. No encontramos diferencias significativas en ninguna de las variables estudiadas. En la prueba Chi Cuadrado se encontró una asociación estadísticamente significativa entre aquellos que tienen estudios superiores finalizados frente a los que no en lo referente a estar trabajando en el sector. Por tanto, se debe seguir potenciando la imagen social de esta etapa ya que los empleadores prefieren dar trabajo a licenciados universitarios con estudios de Formación Profesional que a aquellos que solo tienen estos últimos estudios.

Palabras clave

Formación profesional, imagen social, empleabilidad, Chi Cuadrado, Hostelería y Turismo.

16 Para citar este artículo: Ruiz-Esteban, C. \& González-García, I. (2021). La formación Profesional de hostelería y turismo en la localidad española de Ciudad Real. Informes Psicológicos, 21(1), 247-257 http://dx.doi.org/10.18566/infpsic.v21n1a16 


\section{The Professional Training of hospitality and tourism in the Spanish town of Ciudad Real}

Abstract

Despite the fundamental role that the Spanish educational reform grants to Professional Training, it is unknown whether the perception of society has improved. In order to try to improve the social image of Professional Training, we must get to know it. Although research is scarce, there are some studies that highlight the positive assessment that society gives to the link between training and the company in this type of education. In this sense, the objective of this study is to assess the perception of Professional Training students about the future expectations offered by this formative stage. The participants were 181 Professional Training students in the Hospitality and Tourism Specialty (41.7\% were male). The Future Time Perspective Questionnaire by Husman and Shell (2008) and an ad hoc questionnaire were used to collect sociodemographic data. Student's t tests were performed to analyze the differences in means between different sociodemographic variables in the four dimensions of the questionnaire. No significant differences in any of the variables studied were found. In the Chi-square test, a statistically significant association was found between those who have completed higher education versus those who have not, in relation to working in the sector. Therefore, the social image of this stage should continue to be strengthened, since employers prefer to employ university graduates with Professional Training studies than those who only have these last studies.

Keywords

Professional Training, social image, employability, Chi-square, hospitality and tourism.

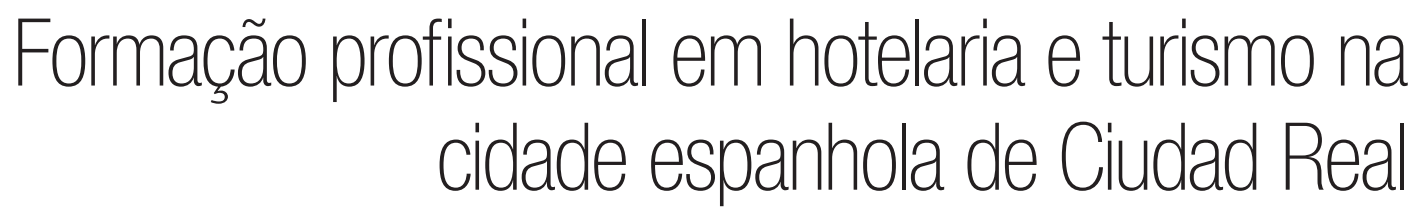

Resumo

Apesar do papel fundamental que a reforma educacional espanhola confere à Formação Profissional, não se sabe se a percepção da sociedade melhorou. Para tentar melhorar a imagem social da Formação Profissional, devemos conhecêla. Embora as pesquisas sejam escassas, existem alguns estudos que destacam a avaliação positiva que a sociedade dá à ligação entre a formação e a empresa nesta modalidade de ensino. Nesse sentido, o objetivo deste estudo é avaliar a percepção de alunos da Formação Profissional sobre as expectativas futuras oferecidas por essa fase formativa. Participaram 181 alunos da Formação Profissional na Especialidade Hotelaria e Turismo (41,7\% do sexo masculino). Foi usado o questionário de perspectivas de futuro de Husman e Shell (2008) e um questionário criado ad hoc para coletar dados sociodemográficos. Testes $t$ de Student foram realizados para analisar as diferenças de médias entre as diferentes variáveis sociodemográficas nas quatro dimensões do questionário. Não encontramos diferenças significativas em nenhuma das variáveis estudadas. No teste Qui Quadrado, foi encontrada associação estatisticamente significativa entre os que possuem o ensino superior completo versus os que não o possuem, em relação ao trabalho no setor. Portanto, a imagem social desta etapa deve continuar a ser fortalecida, uma vez que os empregadores preferem empregar graduados universitários com estudos de formação profissional mais do que aqueles que possuem apenas estes últimos estudos.

Palavras chave

Formação Profissional, imagem social, empregabilidade, Chi Quadrado, hotelaria e turismo. 


\section{ntroducción}

La LOMCE, Ley Orgánica 8/2013, otorga un papel fundamental a la Formación Profesional en la reforma del sistema educativo ya que tiene por finalidad preparar al alumnado para la actividad en un campo profesional y facilitar su adaptación a las modificaciones laborales que pueden producirse a lo largo de su vida, "y permitir su progresión en el sistema educativo y en el sistema de formación profesional para el empleo, así como el aprendizaje a lo largo de la vida" (LOMCE, 2013, p. 30).

Sin embargo, la percepción que la sociedad tiene sobre la Formación Profesional desde su inicio con la Ley General de Educación (1970), y aunque su intención inicial fue potenciar la economía estatal, es la de una educación de segundo nivel comparada con el bachillerato (González, Cueto \& Mato, 2006; Homs, 2008; Merino, 2004) debido a las grandes limitaciones de su implementación (González et al., 2006). Merino y Llosada (2007) señalan que la percepción social de la Formación Profesional mejora con la implantación de la Ley General de Ordenación del Sistema Educativo (LOGSE, 1990). A partir de este momento se generan distintas legislaciones para intentar revalorizar la imagen de estas enseñanzas. Surge así la Ley Orgánica 5/2002, de 19 de junio, de las Cualificaciones y la Formación Profesional (LOCFP), que permitió la realización de cambios en la Formación Profesional, los cuales se materializaron en la aprobación de la Ley Orgánica de Educación (LOE) (2006) y el Real Decreto por el que se creó el Sistema de Formación
Profesional para el Empleo (2007), el cual supuso la integración del Sistema de Formación Ocupacional y el Sistema de Formación Continua.

Los esfuerzos de los diferentes gobiernos españoles por intentar revalorizar la percepción de la sociedad sobre estos estudios se deben a la presión de la OCDE (Organización para la Cooperación y el Desarrollo Económico) ya que España era uno de los países con menores tasas de estudiantes en Formación Profesional (Casquero, 2009; Merino \& Llosada, 2007). Sin embargo, la OCDE estimó que para el año 2020 alrededor de dos tercios del crecimiento del empleo español esté ocupado por técnicos titulados en Formación Profesional. Dichos esfuerzos en las políticas nacionales han tenido su correlato en las políticas de empleo europeas (Sotés-Elizalde, 2009). El Proceso de Copenhague (2002) marca objetivos y prioridades revisables que deben concretarse para cada país, lo que implica un hito en la cooperación europea en materia de Formación Profesional (Renés \& Castro, 2013). En esas prioridades España se compromete a fomentar la excelencia y la calidad de estas enseñanzas y procurará la adecuación entre la formación profesional y el mercado laboral. Además, pretende incrementar el número de titulados de Formación Profesional a través de la potenciación de la imagen social de estos estudios, tal y como sucede en otros países europeos (Pérez \& Rahona, 2009) y de flexibilizar el intercambio entre los distintos niveles educativos en la línea de lo que indica Sanz (2010) al plantear la necesidad de mejorar la orientación en esta etapa educativa.

Una de las razones principales de esta imagen negativa se sitúa en que la 
formación profesional se ha utilizado históricamente para diferentes propósitos. En los años 70 la educación vocacional se utilizó como una opción para las personas de bajo rendimiento (Marhuenda-Fluixá, Salvà, Navas-Saurin, \& Abiétar-López, 2015). Superar esa imagen no está siendo fácil pese a los esfuerzos que en este sentido se han realizado en los últimos años.

Para poder intentar mejorar la imagen social de la Formación Profesional debemos conocerla. Sin embargo, aunque son escasos existen algunos estudios que analizan la percepción social de esta etapa bien centrándose en el profesorado (Agraso, Sánchez, \& Cerqueiras, 2015; Merino, 2003) (Arana, 2010; Casares, 2007; Ferrán, 2012; Merino \& Llosada, 2007; Prieto, 2013; Renés \& Castro, 2013; West \& Mujis, 2008; Zurita, 2006). De ellos destacan las investigaciones de Casares (2007), Renés y Castro (2013) y Zurita (2006), centradas en los estudiantes, donde se destaca positivamente la vinculación entre formación y empresa de este tipo de formación.

Por ello, el objetivo de este estudio es valorar la percepción de los estudiantes de formación profesional sobre las expectativas de futuro que les ofrece esta etapa formativa.

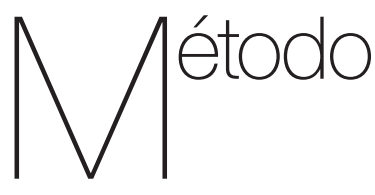

\section{Participantes}

Los participantes en el estudio fueron 181 alumnos (el 58.3\% son mujeres) de Formación Profesional de la rama profesional de Hostelería y Turismo de dos Institutos de Educación Secundaria de Castilla la-Mancha, España. De ellos un $52.2 \%$ se encontraban cursando el primer año del ciclo formativo, mientras que el $47.8 \%$ se encontraban cursando el segundo año. Un $77.2 \%$ de los encuestados y encuestadas cursan sus estudios en el turno diurno. La muestra fue incidental. Los criterios de inclusión fueron: a) estar matriculado en Formación Profesional; b) cursar segundo curso; c) cursar la especialidad de Hostelería o la especialidad de Turismo; y d) dar el consentimiento informado a su participación en la investigación. La población potencial que cumplía los criterios de inclusión eran 198; de ellos, 25 declinaron participar en el estudio y dos fueron excluidos por no contestar a ambos cuestionarios.

\section{Instrumentos}

Para recoger los datos sociodemográficos de los participantes se confeccionó un cuestionario ad hoc para este estudio. Las variables estudiadas en el cuestionario sociodemográfico fueron nueve: Sexo, Edad, Tipo de entorno en que reside (rural, semirural, urbano), Estudios finalizados, Estudios que cursa actualmente, Curso, Turno de estudios, Trabaja en el ámbito de Hostelería y Turismo, Motivos para estudiar un ciclo formativo de Hostelería y Turismo.

Para valorar la percepción de las expectativas que estos estudios les ofrecen, se utilizó el Cuestionario de Perspectivas de Futuro de Husman y Shell (2008). Se trata de una escala Likert de 3 niveles que consta de 22 ítems que se agrupan en 4 dimensiones: integración, valor, extensión y velocidad. 
La dimensión integración se refiere a la tendencia de la persona a vincular el presente y el futuro, de manera que permite al individuo percibir que sus acciones inmediatas constituyen un paso para alcanzar una meta futura (De Volder \& Lens, 1982) (ej. de ítem: Mi visión de futuro es importante en la selección de las acciones que realizo ahora). La segunda dimensión, el valor, corresponde al deseo de sacrificar el presente en beneficio del futuro. Al darle importancia al paso del tiempo, la persona muestra una forma de afecto hacia lo que el futuro puede traerle (Thiébaut, 1998) (Ej de ítem: Encuentro más importante ser quien quiero ser en el futuro que ser quien quiero ser ahora mismo). La extensión es la tercera dimensión de la percepción de expectativa de futuro, y corresponde a la extensión del espacio de tiempo (Nuttin, 1980) de manera que las personas con una amplia representación del futuro son capaces de percibir objetivos distantes en el tiempo como más cercanos y, al hacerlo, esas personas están en mejores condiciones que otras para anticipar más fácilmente las consecuencias a largo plazo de las acciones inmediatas (Tucker, Vuchinich \& Rippens, 2002) (ej. de ítem: En general, seis meses me parecen un período de tiempo muy corto). Finalmente, la cuarta y última dimensión se refiere a la velocidad. Representa la percepción subjetiva del tiempo, no se trata solo de la percepción de las duraciones, sino más en general de la comprensión del paso del tiempo, de la percepción del ritmo al que pasa el tiempo (Husman \& Shell, 2008). A una persona que no está orientada hacia el futuro le resulta difícil organizar actividades futuras ya que el tiempo pasa subjetivamente demasiado rápido. Por el contrario, una persona orientada hacia el futuro generalmente percibe que el tiempo pasa lentamente, por lo que parece apropiado planificar actividades que se llevarán a cabo a largo plazo (ej. de ítem: Siempre siento que estoy haciendo las cosas en el último momento).

El alfa de Cronbach de las cuatro dimensiones es .85, .69, .72 y .53 respectivamente.

\section{Procedimiento}

Se trata de un estudio descriptivo transversal. Tras la selección del IES, se llevó a cabo una entrevista con el equipo de dirección para exponer los objetivos del estudio y detallar los instrumentos así como para solicitar el consentimiento informado. Los participantes fueron informados de la confidencialidad, anonimato y del carácter voluntario del estudio. Los criterios de inclusión fueron los siguientes: estudiantes matriculados en un centro educativo en las ramas de Hostelería o Turismo en los niveles de primer y segundo ciclo de Formación Profesional, firmar el consentimiento informado y que asistiesen a clase en la sesión de administración de los instrumentos de medida. Los motivos de exclusión fueron: dificultades para cumplimentar el instrumento por motivos de idioma, que por alguna discapacidad no fuese posible cumplimentar el instrumento, no haber asistido a clase o que hubiesen sido expulsados del centro educativo coincidiendo con la sesión de administración del instrumento de evaluación.

Todos los participantes firmaron el consentimiento informado tras conocer los objetivos del estudio. Se les aplicaron los cuestionarios a los estudiantes en una única sesión. El análisis de los datos se realizó a través del programa estadístico SPSS. 
Los protocolos del estudio fueron aprobados por el Comité de Ética para Investigaciones Clínicas de la Universidad de Murcia en enero de 2017. Además, el estudio se realizó de acuerdo con las directrices aprobadas según la Declaración de Helsinki.

\section{Análisis de datos}

El tratamiento estadístico de los datos se realizó a través del paquete estadístico SPSS (versión 24.0). Tras la comprobación de los supuestos paramétricos se utilizaron técnicas descriptivas (frecuencias, porcentajes, media y desviación típica). Para valorar las diferencias de medias se utilizó la t de Student. Por último, se utilizaron tablas de contingencia utilizando la prueba Chi-Cuadrado de Pearson para analizar las variables categóricas del estudio. Se comprobaron que se cumplían las condiciones de validez en cada una de las pruebas. Asimismo, para determinar el tamaño del efecto se utilizó el coeficiente $\checkmark$ de Cramer en la prueba Chi-Cuadrado de Pearson.

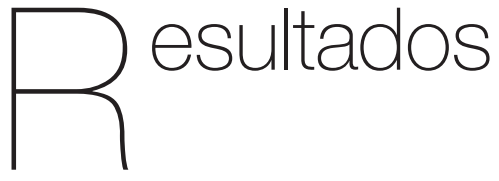

En primer lugar se realizó un estudio porcentual de las variables sociodemográficas. Entre los motivos por los que estudian Formación Profesional, un 33.9\% indicó que consideran que es la mejor opción para trabajar, un 42.8\% porque les gusta la profesión y tan solo un 23.3\% porque no les gusta estudiar. Pero, sin duda, resulta llamativo que un $9.3 \%$ de los estudiantes cursaran previamente estudios superiores antes de matricularse en los estudios de Hostelería y Turismo, un $27.2 \%$ accede a través de Bachillerato y el resto a través de Educación Secundaria Obligatoria-ESO (34.5\%) o Programas de Garantía Social (30\%). Por ello, se realizó un Análisis de Varianza para establecer las posibles diferencias entre las dimensiones según los estudios previos sin obtener resultados significativos.

Se llevaron a cabo t de Student para analizar las diferencias de medias entre distintas variables sociodemográficas en las cuatro dimensiones del cuestionario. No encontramos diferencias significativas por sexo (Integración: $p=.475$; Valor: $p=$ .957; Extensión: $p=.990$; y Velocidad: $p$ $=.142$ ), ni por curso (Integración: $p=.480$; Valor: $p=.287$; Extensión: $p=.674$; y Velocidad: $p=.451)$. Sin embargo, sí existía una diferencia significativa en el turno diurno o nocturno y la dimensión Valor (ver Tabla 1).

Tabla 1.

Diferencia de medias por turno

\begin{tabular}{|c|c|c|c|c|c|c|}
\hline & & $\mathrm{N}$ & Media & $\begin{array}{c}\text { Desviación } \\
\text { Típica }\end{array}$ & gl & $p$ \\
\hline \multirow{2}{*}{ 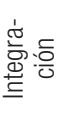 } & Nocturno & 41 & 5.487 & 1.075 & \multirow{2}{*}{176} & \multirow{2}{*}{.830} \\
\hline & Diurno & 139 & 5.446 & 1.097 & & \\
\hline \multirow{2}{*}{$\frac{\overline{0}}{\frac{\pi}{7}}$} & Nocturno & 41 & 4.414 & 1.532 & \multirow{2}{*}{176} & \multirow{2}{*}{.050} \\
\hline & Diurno & 139 & 3.884 & 1.619 & & \\
\hline \multirow{2}{*}{ 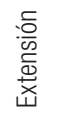 } & Nocturno & 41 & 2.390 & .891 & \multirow{2}{*}{176} & \multirow{2}{*}{.098} \\
\hline & Diurno & 139 & 2.093 & 1.034 & & \\
\hline \multirow{2}{*}{$\begin{array}{l}\text { 옹 } \\
\frac{0}{0} \\
\frac{0}{0} \\
>\end{array}$} & Nocturno & 41 & 1.000 & .774 & \multirow{2}{*}{176} & \multirow{2}{*}{.669} \\
\hline & Diurno & 139 & 0.942 & .749 & & \\
\hline
\end{tabular}


Por último, se realizó una Prueba Chi cuadrado (ver Tabla 2). Esta prueba indicaba que entre los participantes del estudio apareció una asociación estadísticamente significativa entre aquellos que tienen estudios superiores finalizados frente a los que no en cuanto a estar trabajando en Turismo y Hostelería $(V=$ $.033 ; p=.040)$.

Tabla 2.

Estudios Superiores finalizados y estar trabajando en el sector del Turismo y la hostelería.

\begin{tabular}{|c|c|c|c|c|c|c|}
\hline \multicolumn{2}{|c|}{ Estudios finalizados/Trabajo en el sector } & \multirow{2}{*}{$\begin{array}{c}\mathbf{S i} \\
32(33 \%)\end{array}$} & \multirow{2}{*}{$\begin{array}{c}\text { No } \\
30(36.1 \%)\end{array}$} & \multirow{2}{*}{$\chi^{2}$} & \multirow[t]{2}{*}{$\mathrm{p}$} & \multirow[t]{2}{*}{$\sqrt{ }$} \\
\hline \multirow{5}{*}{ Estudios finalizados } & ESO & & & & & \\
\hline & CFGS/CFGM & $37(38.1 \%)$ & $17(20.5 \%)$ & \multirow{4}{*}{1.466} & \multirow{4}{*}{.040} & \multirow{4}{*}{.603} \\
\hline & & & & & & \\
\hline & Bachiller & $20(20.6 \%)$ & $29(34.9 \%)$ & & & \\
\hline & Universitarios & $8(8.4 \%)$ & $7(8.2 \%)$ & & & \\
\hline
\end{tabular}

Nota: ESO: Educación Secundaria Obligatoria; CFGS/CFGM: ciclos formativos de grado superior/ciclos formativos de grado medio

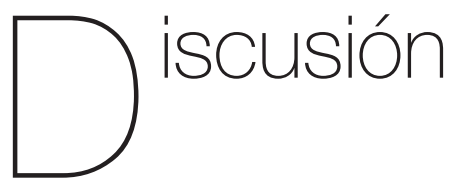

Aunque la formación profesional ha tenido una imagen negativa en el pasado que se ha ido restituyendo con el tiempo, hoy todavía existen resquicios de ese desprestigio y muchos estudiantes que fracasan en la enseñanza obligatoria terminan en la Formación Profesional con el fin de conseguir una titulación oficial que posibilite su inserción laboral. Los empresarios cada vez demandan más titulados de ciclos formativos y la tasa de inserción de estos estudios es mayor que la de otros, lo que contribuye a esta mejora de imagen.

Las investigadores coinciden en que la percepción social de los estudios de Formación Profesional mejora entre los implicados, tanto profesores, empleadores como estudiantes (Agraso et al., 2015; Merino, 2003; 2004; 2007). Centrándonos en la imagen percibida por los estudiantes, mientras hace unos años se elegían estos estudios como segunda opción, en la actualidad la gran mayoría de los que estudian Formación Profesional en la rama de Turismo y Hostelería lo hacen, bien porque creen que es la mejor opción para una mayor empleabilidad o bien porque les gusta la profesión. Hay un porcentaje cercano al $25 \%$ que lo hacen porque no les gusta estudiar. Es de resaltar que un $8.3 \%$ son estudiantes que han terminado estudios superiores y vuelven a cursar estudios de formación profesional ya que consideran que son la mejor opción para formarse y trabajar en este ámbito. Estos resultados son coherentes con la autopercepción positiva que muestran los estudiantes en los trabajos de Renés y Castro (2013) y Zurita (2003). Es además un síntoma claro de que va mejorando la orientación hacia esta etapa educativa, en la línea 
de lo expuesto por Sanz (2010), cuando un porcentaje de la población considera que la empleabilidad de esta etapa es mejor que la de poseer estudios universitarios, que es lo que subyace a la elección de estos estudios con posterioridad a la graduación universitaria.

Los estudios de Formación Profesional son considerados por los estudiantes y sus familias como una opción válida, ya sea para obtener una cualificación que posibilite su acceso al mundo laboral o como paso intermedio a la Universidad. Según Agraso et al. (2015) aunque los matriculados en este tipo de enseñanzas todavía es menor que las tasas de matrícula en titulaciones universitarias, la diferencia se reduce año tras año.

Sin embargo, y a pesar de la buena percepción que muestran los implicados, todavía se debe potenciar la imagen social de esta etapa ya que los empleadores prefieren dar trabajo a licenciados universitarios con estudios de Formación Profesional que a aquellos que solo tienen estos últimos estudios. Por tanto, debemos seguir las indicaciones de Pérez y Rahona (2009) y seguir potenciando la imagen de esta etapa educativa para incrementar el número de titulados.

Uno de los grandes problemas de la educación vocacional ha sido provocado, tal como indican Marhuenda-Fluixá et al. (2015), porque ha sido vista como la opción natural para los alumnos de bajo rendimiento a una edad temprana; lo que alguna vez fue una elección excepcional se ha convertido en la medida estándar. La unión de estudiantes de Formación Profesional convencidos de la opción educativa elegida con aquellos a los que el sistema deriva por bajo rendimiento genera una mayor tasa de abandono y la pobre imagen social a la que se alude en este estudio. Sin embargo, la tasa de empleabilidad de los que realmente eligen esta opción libremente sigue incrementándose según datos del Instituto Nacional de Estadística (INE, 2018).

Los resultados de este estudio pueden ser de gran utilidad en la orientación profesional y laboral de los estudiantes. Además, resulta de gran importancia que la familia conozca e inculque en las salidas profesionales de la formación profesional.

Entre las limitaciones de este estudio se encuentra el hecho de que se trata de un estudio transversal, así como el hecho de trabajar con una muestra incidental no representativa y la utilización de medidas de autoinforme que pueden estar influidas por la deseabilidad social.

Sería deseable como prospectiva que se plantearan estudios longitudinales no solo de los implicados en la Formación Profesional sino a nivel de todos los estamentos de la sociedad para conocer en detalle la percepción que tiene la ciudadanía de estos estudios. Sería interesante incluir variables como tasas de empleabilidad, traspaso de estudiantes entre niveles educativos (universidad y formación profesional), valoración de la Formación Profesional por sectores profesionales, estudio de salarios y de desarrollo profesional, etc. Todavía queda mucho trabajo institucional por realizar para revalorizar esta etapa educativa cada vez más demandada por el mercado. 


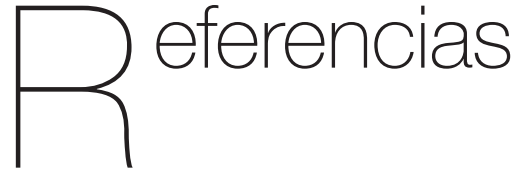

Agraso, L. R., Sánchez, A. F. R., \& Cerqueiras, E. M. B. (2015). La formación en alternancia en la universidad y en los ciclos formativos: aportaciones desde dos investigaciones realizadas en Galicia. Educar, 51(2), 349-371.

Arana, J. (2010). La formación ocupacional por competencias y su certificabilidad y convalidación con la Formación Profesional Reglada. (Tesis de Método de Investigación y Diagnóstico en Educación), Universidad de Granada, España. Recuperado de http://hdl.handle.net/10481/15081

Casares, E. (2007). Estudio descriptivo de los programas de garantía social de iniciación profesional en los centros de Granada. (Tesis de Didáctica y Organización Escolar), Universidad de Granada, España. Recuperado de http://hdl.handle. net/10481/1662

Casquero, A. (2009). Demanda e inserción laboral de la Formación Profesional Reglada en España. Revista del Instituto de Estudios Económicos, 2, 213-245.

Comisión Europea (2002). Proceso de Copenhague: una cooperación europea reforzada en materia de educación y formación profesionales. Recuperado de http://europa.eu/legislation summaries/education_training_youth/ vocational_training/ef0018_es.htm

De Volder, M. L., \& Lens, W. (1982). Academic achievement and future time perspective as a cognitive-motivational concept. Journal of Personality and Social Psychology, 42(3), 566.

Ferrán, C. M. (2012). Percepciones de distintos agentes sociales acerca de la educación formal de los chicos y chica tutelados. Educatio Siglo XXI, 30(2), 185-206.

García-Jiménez, E., \& Lorente-García, R. (2015). Recorrido por la imagen social de la formación profesional: un camino hacia su revalorización. Revista española de educación comparada, 26, 119-134.

González, M. C., Cueto, B., \& Mato, J. (2006). El papel de la formación en centros de trabajo en la inserción laboral de los titulados. Revista de Educación, 341, 337-372.

Homs, O. (2008). La formación profesional en España: hacia la sociedad del conocimiento. Barcelona: Obra Social La Caixa.

Husman, J., \& Shell, D. F. (2008). Beliefs and perceptions about the future: $A$ measurement of future time perspective. Learning and individual differences, 18(2), 166-175.

Instituto Nacional de Estadística (INE) (2018). Datos de empleabilidad por nivel educativo (2015-2017). Recuperado de http:/www. ine.es/

Ley Orgánica 10/2002, de 23 de diciembre, de Calidad de la Educación. BOE núm. 307 de 24 de Diciembre de 2002. Referencia: BOE-A-2002-25037.

Ley Orgánica 2/2006, de 3 de mayo, de Educación. «BOE» núm. 106, de 4 de mayo de 2006, páginas 17158 a 17207 (50 págs.) 
Ley Orgánica 8/2013, de 9 de diciembre, para la mejora de la calidad educativa. "BOE» núm. 295, de 10 de diciembre de 2013 Referencia: BOE-A-2013-12886

Marhuenda-Fluixá, F., Salvà, F., Navas-Saurin, A. A., \& Abiétar-López, M. (2015). Twenty years of basic vocational education provision in Spain. Changes and trends. International journal for research in vocational education and training, 2(2), 137-151.

Merino, R. (2003). Luces y sombras de la reforma de la formación profesional: la visión de los profesores de enseñanza secundaria. Aula Abierta, 82, 5-26.

Merino, R. (2004). De la contrarreforma de la formación profesional en la LGE a la contrarreforma de la LOGSE. Flujos y estrategias de los alumnos en la secundaria postobligatoria. En B. Mulet (Coord). Sociologia de l'educació: cultura i práctica escolar: les reformes actuals (pp. 611-620). Islas Baleares: Universitat de les Illes Balears.

Merino, R. \& Llosada, J. (2007). ¿Puede una reforma hacer que más jóvenes escojan formación profesional? Flujos e itinerarios de formación profesional de los jóvenes españoles. Tempora, 10, 215-244.

Ministerio de Educación y Formación Profesional (2019). Panorama de la educación 2 Indicadores de la OCDE 2019. Informe español. Recuperado de https://www.educacionyfp.gob.es/ dam/jcr:b8f3deec-3fda-4622-befb386a4681b299/panorama\%20de\%20 la\%20educaci\%C3\%B3n\%202019.pdf
Nuttin, J. (1980). Motivation et perspectives d'avenir (Vol. 14). Presses universitaires de Louvain: Lovaina, Bélgica.

Pérez, C. y Rahona, M. (2009). La formación profesional en España y el mercado de trabajo: desafíos y oportunidades. En XVI Encuentro de Economía Pública: 5 y 6 de febrero de 2009: Palacio de Congresos de Granada

Prieto, S. C. (2013). La formación profesional de grado medio en México y España: un estudio comparado. (Tesis de Didáctica y Teoría de la Educación). Universidad Autónoma de Madrid, España. Recuperado de http://digitool-uam.greendata.es:1801/ webclient/DeliveryManager?pid=60252\&c ustom_att_2=simple_viewer

Renés, P. \& Castro, A. (2013). Análisis de la situación de la formación profesional desde el punto de vista de sus protagonistas. Educatio Siglo XXI, 31(2), 255-276.

Sanz, C. (2010). La orientación profesional en los sistemas de formación profesional. REOP, 21(3), 643-652.

Sotés-Elizalde, M. A. (2009). Formación profesional: sistema educativo y empresa. Educación y Educadores, 12(1), 109-118.

Thiébaut, E. (1998). La perspective temporelle, un concept a la recherche d'une définition opérationnelle. L'Année Psychologie, 98, 101-125.

Tucker, J. A., Vuchinich, R. E., \& Rippens, P. D. (2002). Predicting natural resolution of alcohol-related problems: A prospective behavioral economic analysis. Experimental and Clinical Psychopharmacology, 10(3), 248. 
West, M. \& Mujis, D. (2008). Personalized learning. In C. Chapman \& H. Hunter (Eds.), Radical reform, (pp. 128-143). Londres: Routledge.
Zurita, F. J. (2006). Análisis descriptivo de la formación en centros de trabajo (FCT) de familias profesionales técnico-industriales en el ámbito provincial de Zaragoza. (Tesis de Pedagogía). Universitat Rovira i Virgili, España. Recuperado de http://hdll.handle. net/10803/8910 